\title{
Predictive Response Value of Pre- and Postchemoradiotherapy Variables in Rectal Cancer: An Analysis of Histological Data
}

\author{
Marisa D. Santos, ${ }^{1}$ Cristina Silva, ${ }^{1}$ Anabela Rocha, ${ }^{1}$ Carlos Nogueira, ${ }^{1}$ \\ Eduarda Matos, ${ }^{2}$ and Carlos Lopes ${ }^{3,4}$ \\ ${ }^{1}$ Department of Surgery, Digestive Surgery Service, Hospital de Santo António, Centro Hospitalar do Porto, \\ Largo Abel Salazar, 4099-003 Porto, Portugal \\ ${ }^{2}$ Department of Community Health, Instituto de Ciências Biomédicas Abel Salazar, Rua Jorge Viterbo Ferreira, \\ No. 228, 4050-313 Porto, Portugal \\ ${ }^{3}$ Department of Pathology, Pathological Anatomy Service, Hospital de Santo António, Centro Hospitalar do Porto, \\ Largo Professor Abel Salazar, 4099-003 Porto, Portugal \\ ${ }^{4}$ Department of Pathology and Molecular Immunology, Instituto de Ciências Biomédicas Abel Salazar, \\ Rua Jorge Viterbo Ferreira, No. 228, 4050-313 Porto, Portugal
}

Correspondence should be addressed to Marisa D. Santos; marisadsantos@gmail.com

Received 29 July 2015; Accepted 30 September 2015

Academic Editor: Shahid Pervez

Copyright (C) 2016 Marisa D. Santos et al. This is an open access article distributed under the Creative Commons Attribution License, which permits unrestricted use, distribution, and reproduction in any medium, provided the original work is properly cited.

Background. Neoadjuvant chemoradiotherapy (nCRT) followed by curative surgery in locally advanced rectal cancer (LARC) improves pelvic disease control. Survival improvement is achieved only if pathological response occurs. Mandard tumor regression grade (TRG) proved to be a valid system to measure nCRT response. Potential predictive factors for Mandard response are analyzed. Materials and Methods. 167 patients with LARC were treated with nCRT and curative surgery. Tumor biopsies and surgical specimens were reviewed and analyzed regarding mitotic count, necrosis, desmoplastic reaction, and inflammatory infiltration grade. Surgical specimens were classified according to Mandard TRG. The patients were divided as "good responders" (Mandard TRG1-2) and "bad responders" (Mandard TRG3-5). According to results from our previous data, good responders have better prognosis than bad responders. We examined predictive factors for Mandard response and performed statistical analysis. Results. In univariate analysis, distance from anal verge and ten other postoperative variables related with nCRT tumor response had predictive value for Mandard response. In multivariable analysis only mitotic count, necrosis, and differentiation grade in surgical specimen had predictive value. Conclusions. There is a lack of clinical and pathological preoperative variables able to predict Mandard response. Only postoperative pathological parameters related with nCRT response have predictive value.

\section{Introduction}

Neoadjuvant chemoradiotherapy (CRT) followed by total mesorectum excision (TME) surgery and systemic chemotherapy remains the standard of care for locally advanced rectal cancer (LARC), but only a few patients benefit from this treatment modality. Sauer et al. [1] reported a persistent significant improvement of preoperative CRT versus postoperative CRT on local control, although without overall survival improvement, which is only achieved in situations of good or complete pathological response to CRT
$[2,3]$. Chemoradiation induces tumor downstaging effect, which increases the probability of a complete resection and a sphincter- preserving surgery, benefiting local control. However, some patients still develop distant and/or local recurrence that compromise survival, particularly those with poor pathological tumor response. The histological response to preoperative CRT has been reported in several studies to be closely related to oncologic outcomes [2, 4-7]. A complete pathologic response (pCR), which is characterized by sterilization of all tumor cells, leads to excellent prognosis and is observed in approximately $10 \%$ to $30 \%$ of cases [8]. 
The remaining patients exhibit a spectrum of residual disease, ranging from microscopic tumor cell foci on a background of radiation-induced fibrosis to no response at all [9]. The prognostic relevance of pathological tumor response according to several standard grading criteria has been studied extensively $[4,10-13]$. In our previous study, the Mandard system proved to be a good determinant of outcome, when cases were grouped into TRG1+2 (good responders) and TRG3+4+5 (bad responders). This methodology proved superior compared to division in groups of ypCR (TRG1) versus all other (TRG2-5) regarding disease-free survival (DFS) and overall survival (OS) [14]. For this reason it would be of great value to predict nCRT-induced histologic regression and tumor shrinkage. The ability to predict the response, either before treatment or during its early stages, could spare "bad responders" patients the expense and stress of undergoing treatment from which they will derive no benefit. Instead, these patients would be candidates for more intensive treatment strategies. Identifying "bad responders" only in posttreatment may also be an indicator for a different and more intensive adjuvant therapy. Nowadays, individualizing the treatment approach in LARC is a demanding goal to achieve.

The present study aims to analyze predictive factors of Mandard tumor response, trying to identify and characterize the "good" and the "bad responders," comparing classic histological data obtained from diagnostic biopsies with histological alterations from surgical specimens after treatment.

\section{Materials and Methods}

2.1. Patient Population. A single-institution database was queried for consecutive patients with LARC, biopsy-proven rectal adenocarcinoma, who underwent nCRT followed by elective radical surgery with TME with curative intent between January 1, 2003, and December 31, 2013.

Admission Criteria. Patients with rectal cancers located at less than $12 \mathrm{~cm}$ of distance from the anal verge and clinical stages cT2N+M0 or cT3/4 N0/+M0 are included.

Exclusion Criteria. Patients with other diagnosed neoplasia, short course RT, yp stage IV, R1/R2 surgery, and death within 60-day postoperative time are excluded.

All patients receiving nCRT who were operated within 8 weeks after radiotherapy conclusion were included in this analysis. Patients receiving short-course radiation were excluded since no downstaging occurs when immediate surgery is carried out.

Staging included rigid proctoscopy, total colonoscopy, chest, abdominal, and pelvic CT scan, endorectal ultrasound (ERUS), pelvic magnetic resonance image (MRI) (since 2008), and carcinoembryonic antigen (CEA) serum levels.

Diagnostic pretreatment paraffin-embedded biopsies of 152 patients (91\%) were available and reviewed by an independent element. All of them were characterized in terms of grade, mitotic index (for 10 fields), necrosis grade (scarce/moderate/marked), inflammatory reaction (scarce/moderate/marked), and desmoplastic infiltration (scarce, moderate, and marked). Tumor biopsy samples classification, including grade, were obtained on worst areas whenever available material contained several areas with neoplasia.

Neoadjuvant CRT protocol included total irradiation of 50.4 Gy in 28 fractions and 5-fluorouracil by infusion pump.

Radical surgery consisted mainly in sphincter saving rectal resection (SSRE) or abdominoperineal resection (APR) both with TME. Regarding operative procedure selection, we considered the distance of the lesion to the anus, the comorbidities of the patient, and the condition of the anal sphincter.

The number of samples taken from the resected specimens was variable, with a mean of 6 paraffin blocks per case. The methodology used was the following:

(1) Five samples were from the area with macroscopic lesion (assuming it exists), that is, the same as dealing with a specimen from a patient who has not received neoadjuvant therapy. These included the closest macroscopic approach of the macroscopic lesion to the peritoneal surface and/or the mesorectal excision plane, as appropriate.

(2) If no viable tumour was identified within the initial 5 blocks, the whole of the remainder of any macroscopic lesion in additional blocks was included.

(3) If no viable tumour was identified within the initial or extra blocks, another three further levels from all of these blocks were taken. If no viable tumour was identified in these sections then complete histological tumour regression was assumed.

All obtained slides were seen and reviewed by the same pathologist. Items observed and registered in the biopsies were subsequently analyzed in the resected specimen and the same criteria adopted.

Standard pathologic tumor staging of the resected specimen was performed in accordance with the guidelines of the American Joint Committee on Cancer (AJCC). Circumferential resection margin (CRM) was scored as positive when cancer cells were within $1 \mathrm{~mm}$ of the margin. Evidence of ypCR was defined as an absence of viable adenocarcinoma in the surgical specimen or the presence of lakes of mucus without tumor cells.

The histology of all surgical specimens was reviewed and confirmed by an independent element and were classified based on Mandard tumor regression grading system.

Patients were divided in two groups according to Mandard TRG system: good responders were defined as Mandard TRG1 or TRG2; bad responders were defined as Mandard TRG3, TRG4, or TRG5 (Figure 1).

Both groups (good responders versus bad responders) were used to evaluate outcome results. Operated patients were subjected to adjuvant chemotherapy protocol for 6 months performed preferably with 5-fluorouracil (5-FU) or a combination 5-FU and oxaliplatinum.

Disease recurrence was evaluated according to location: locoregional (LR), systemic (DR), or mixed. 


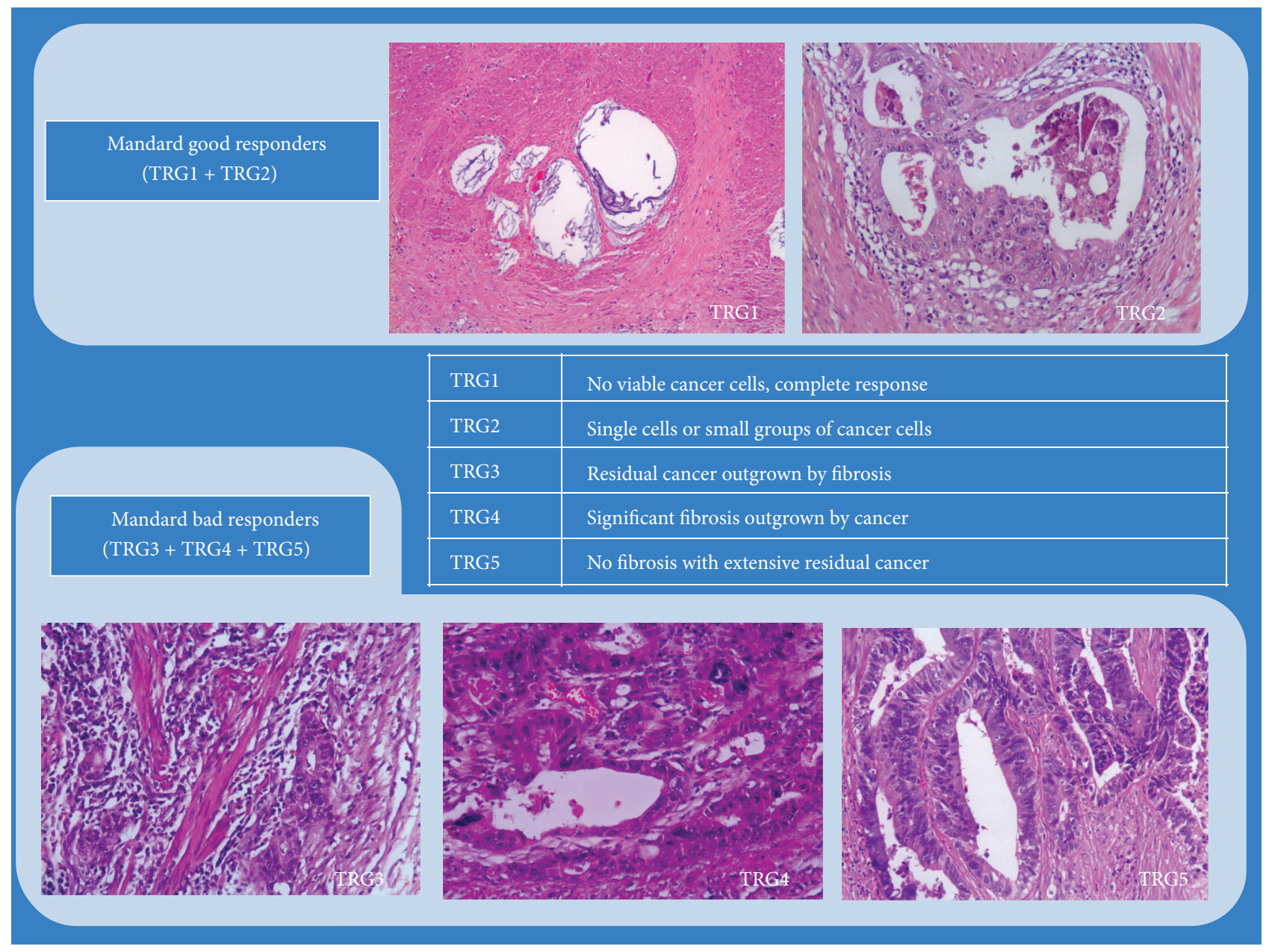

FIgURE 1: Mandard system.

None of the patients were lost for follow-up.

All surviving patients were observed and their current status was confirmed.

Clinical nodal staging may present some inaccuracies; consequently, downstaging evaluation including $\mathrm{N}$ stage may introduce some bias, so the term "downstaging" used to describe the efficacy of treatment was defined specifically as T stage downstaging.

Disease-free survival (DFS) was calculated from the date of surgery to the date of progression (local and/or distant), and overall survival (OS) was calculated from the first date of treatment to the date of death or last follow-up.

\subsection{Statistical Analysis. The survival function was estimated} using the Kaplan-Meier method. The difference in survival rates between groups was tested for significance using the log rank test. The significance of differences in proportions was calculated with Chi-square test and the differences in means with Student's $t$-test. A logistic regression analysis was used to assess the independent significance of factors predictive of response, defined as "good" or "bad" responders (TRG1-2/TRG3-5): age; gender; clinical stage; anal-tumor distance; pretreatment CEA; biopsy specimen characteristics: mitotic index, desmoplastic infiltration, inflammatory reaction, and necrosis; CEA post-CRT; surgical procedure; surgical morbidity; pathological stage; circumferential involvement; tumor grade; tumor mitosis number; tumor desmoplastic infiltration; tumor inflammatory reaction; and tumor necrosis in resected specimen were studied.

The statistical analysis was made with SPSS statistical software (version 21.0 for Windows; SPSS Inc., Chicago, IL). All statistical tests were conducted at a two-sided level of significance of 0.05 .

\section{Results}

3.1. Patient Population. Between January 2003 and December 2013, 186 consecutive patients with LARC were treated with neoadjuvant CRT followed by TME surgery at one single university hospital. We excluded 11 patients with positive radial margin (R1 surgery), 4 patients with yp stage IV, and four deaths within 60 days of postoperative period. In the end, 167 patients were included in the present analysis. Male: female ratio was $1.69: 1$. Median age was 64.6 years (range, 29-83 years). Clinical parameters are summarized in Table 1. 
TABLE 1: Clinical parameters.

\begin{tabular}{|c|c|}
\hline Variable & $N(\%)$ \\
\hline \multicolumn{2}{|l|}{ Age } \\
\hline Mean & 64.62 \\
\hline Range & $(29-83)$ \\
\hline \multicolumn{2}{|l|}{ Gender } \\
\hline Male & $105(62.9)$ \\
\hline Female & $62(37.1)$ \\
\hline \multicolumn{2}{|l|}{ Tumor length } \\
\hline$<4 \mathrm{~cm}$ & $41(24.6)$ \\
\hline$\geq 4 \mathrm{~cm}$ and $<6 \mathrm{~cm}$ & $85(50.8)$ \\
\hline$\geq 6 \mathrm{~cm}$ & $41(24.6)$ \\
\hline \multicolumn{2}{|l|}{ Tumor circumference } \\
\hline$\leq 1 / 3$ & $27(16.2)$ \\
\hline$>1 / 3$ and $\leq 1 / 2$ & $59(35.3)$ \\
\hline$>1 / 2$ and $\leq 2 / 3$ & $39(23.4)$ \\
\hline$>2 / 3$ and $\leq 3 / 3$ & $42(25.1)$ \\
\hline \multicolumn{2}{|l|}{ Distance from anal verge } \\
\hline$>6 \mathrm{~cm}$ & $81(48.5)$ \\
\hline$\leq 6 \mathrm{~cm}$ & $86(51.5)$ \\
\hline \multicolumn{2}{|l|}{ CEA pre-CRT } \\
\hline$<5$ & $116(69.5)$ \\
\hline$\geq 5$ & $44(26.3)$ \\
\hline Missing & $7(4.2)$ \\
\hline \multicolumn{2}{|l|}{ cT stage } \\
\hline $2-3$ & $152(91.0)$ \\
\hline 4 & $15(9.0)$ \\
\hline \multicolumn{2}{|l|}{ Clinical stage } \\
\hline II & $76(45.5)$ \\
\hline III & $91(54.5)$ \\
\hline \multicolumn{2}{|l|}{ CEA post-CRT } \\
\hline$<5$ & $141(84.4)$ \\
\hline$\geq 5$ & $14(8.4)$ \\
\hline Missing & $12(7.2)$ \\
\hline \multicolumn{2}{|l|}{ Surgery procedure } \\
\hline AAR/SSO & $107(64.1)$ \\
\hline AAP/others & $60(35.9)$ \\
\hline \multicolumn{2}{|l|}{ Surgery } \\
\hline Open & $129(77.2)$ \\
\hline Laparoscopic & $38(22.8)$ \\
\hline Perioperative complications & $42(25.1)$ \\
\hline \multicolumn{2}{|l|}{ Morbidity } \\
\hline Abdominal or pelvic abscess & $16(9.5)$ \\
\hline Anastomose leak & $3(1.7)$ \\
\hline Reoperation & $6(3.5)$ \\
\hline Readmission & $3(1.7)$ \\
\hline
\end{tabular}

3.2. Biopsy Characteristics. The biopsy results are shown in Table 2.

The mitotic index is the number of mitoses for 10 highpowered fields and the cut-off was chosen taking into account the best ratio of sensitivity: specificity. If no 10 high-power fields were present with neoplasia, the number of mitoses was
TABLE 2: Biopsies characteristics.

\begin{tabular}{|c|c|}
\hline Variable & $N(\%)$ \\
\hline \multicolumn{2}{|l|}{ Biopsy } \\
\hline \multicolumn{2}{|l|}{ Grade } \\
\hline 1 & $46(27.5)$ \\
\hline 2 & $98(58.7)$ \\
\hline 3 & $8(4.8)$ \\
\hline Missing & $15(9.0)$ \\
\hline \multicolumn{2}{|l|}{ Mucinous presence } \\
\hline No & $145(86.8)$ \\
\hline Yes & $7(4.2)$ \\
\hline Missing/not applied & $15(9.0)$ \\
\hline \multicolumn{2}{|l|}{ Mitosis number } \\
\hline$\leq 9.5$ & $34(20.4)$ \\
\hline$\geq 9.6$ & $117(70.0)$ \\
\hline Missing/not applied & $16(9.6)$ \\
\hline \multicolumn{2}{|l|}{ Inflammatory infiltrate } \\
\hline Scarce & $33(19.8)$ \\
\hline Moderated & $66(39.5)$ \\
\hline Marked & $52(31.1)$ \\
\hline Missing/not applied & $16(9.6)$ \\
\hline \multicolumn{2}{|l|}{ Desmoplastic reaction } \\
\hline Scarce & $44(26.3)$ \\
\hline Moderated & $80(47.9)$ \\
\hline Marked & $27(16.2)$ \\
\hline Missing/not applied & $16(9.6)$ \\
\hline \multicolumn{2}{|l|}{ Necrosis degree } \\
\hline Scarce & $79(47.3)$ \\
\hline Moderated & $40(23.9)$ \\
\hline Marked & $32(19.2)$ \\
\hline Missing/not applied & $16(9.6)$ \\
\hline
\end{tabular}

counted in the number of observed fields, reducing the final result to 10 fields.

3.3. Surgery. Sphincter saving rectal resection with anastomosis (with or without protective ileostomy) was performed on 107 patients (64.1\%). Abdominoperineal resection was performed on 53 patients, and seven patients were subjected to proctectomy with definitive stoma. The perioperative morbidity of the series was of $25,1 \%$ with 16 abdominal or pelvic abscesses, 3 anastomotic leaks, 6 reoperations, and 3 readmissions.

3.4. Pathology. Stage distribution is shown in Table 3.

The average number of dissected lymph nodes in the surgical specimen was 8.2 (range $0-22$ ).

Circumferential resection margin $>1 \mathrm{~mm}$ was confirmed in all 167 patients. Response to neoadjuvant therapy is characterized in Table 3.

Downstaging and Final Pathologic Stage Classification. T stage downstaging was observed in 67 patients (40.1\%). Reduction in $\mathrm{T}$ stage by one level was observed in 29 patients (17.4\%) and 
TABLE 3: Pathological parameters.

\begin{tabular}{|c|c|}
\hline Variable & $N(\%)$ \\
\hline \multicolumn{2}{|l|}{ ypT stage } \\
\hline pT0-1 & $38(22.8)$ \\
\hline pT2-4 & $129(77.2)$ \\
\hline \multicolumn{2}{|l|}{ ypN stage } \\
\hline pN0 & $110(65.9)$ \\
\hline pN1-2 & $57(34.1)$ \\
\hline \multicolumn{2}{|l|}{ Pathological stage } \\
\hline 0 & $58(34.7)$ \\
\hline II & $58(34.7)$ \\
\hline III & $51(30.5)$ \\
\hline \multicolumn{2}{|l|}{ T downstaging } \\
\hline Yes & $67(40.1)$ \\
\hline No & $100(59.9)$ \\
\hline \multicolumn{2}{|l|}{ Pathological TNM downstaging } \\
\hline Yes & $95(56.9)$ \\
\hline No & $72(43.1)$ \\
\hline \multicolumn{2}{|l|}{ CRM distance } \\
\hline$>2 \mathrm{~mm}$ & $159(95.2)$ \\
\hline$\leq 2$ and $>1 \mathrm{~mm}$ & $8(4.8)$ \\
\hline \multicolumn{2}{|l|}{ Mandard TRG } \\
\hline TRG1-2 (good responders) & $85(50.9)$ \\
\hline TRG3-5 (bad responders) & $82(49.1)$ \\
\hline \multicolumn{2}{|c|}{ Other characteristics of resected specimen } \\
\hline \multicolumn{2}{|l|}{ Grade } \\
\hline 0 & $32(19.2)$ \\
\hline 1 & $31(18.6)$ \\
\hline 2 & $98(58.7)$ \\
\hline 3 & $6(3.6)$ \\
\hline \multicolumn{2}{|l|}{ Mucinous presence } \\
\hline Yes & $41(24.5)$ \\
\hline No & $126(75.4)$ \\
\hline \multicolumn{2}{|l|}{ Inflammatory infiltrate } \\
\hline Scarce & $60(35.9)$ \\
\hline Moderated & 75 (44.9) \\
\hline Marked & $28(16.8)$ \\
\hline Missing & $4(2.4)$ \\
\hline \multicolumn{2}{|l|}{ Desmoplastic reaction } \\
\hline Scarce & $45(26.9)$ \\
\hline Moderated & $64(38.3)$ \\
\hline Marked & $51(30.5)$ \\
\hline Missing & $7(4.2)$ \\
\hline \multicolumn{2}{|l|}{ Necrosis grade } \\
\hline Scarce & $27(16.2)$ \\
\hline Moderated & $38(22.8)$ \\
\hline Marked & $99(59.3)$ \\
\hline Missing & $3(1.8)$ \\
\hline \multicolumn{2}{|l|}{ Mitosis number } \\
\hline$\leq 9.5$ & $73(43.7)$ \\
\hline$>9.5$ & $68(40.7)$ \\
\hline Missing & $26(15.6)$ \\
\hline
\end{tabular}

TABLE 3: Continued.

\begin{tabular}{lc}
\hline Variable & $N(\%)$ \\
\hline Lymphatic permeation & $100(59.9)$ \\
$\quad$ No & $67(40.1)$ \\
$\quad$ Yes & \\
Vascular permeation & $142(85)$ \\
$\quad$ No & $25(15)$ \\
Yes & \\
Perineural permeation & $96(57.5)$ \\
No & $71(42.5)$ \\
Yes & \\
Distal margin & $108(65.7)$ \\
$\geq 2 \mathrm{~cm}$ & $59(35.3)$ \\
$<2 \mathrm{~cm}$ and $\geq 1 \mathrm{~cm}$ &
\end{tabular}

by two or more levels in 38 patients (22.8\%). Observations indicating pathologic downstaging are given in Table 3. Ninety-five $(56.8 \%)$ patients presented pathologic stage one or lower pathologic stage than initial clinical tumor stage. Pathologic complete response (ypCR or Mandard TRG1) was confirmed in 31 patients $(18.5 \%)$.

TRG Classification. The use of Mandard system allowed us to define two groups as previously mentioned: good responders (Mandard TRG1-2) and bad responders (Mandard TRG35). Using Mandard system a good response to nCRT was attributed to 85 patients $(50.9 \%)$ and a bad response was attributed to 82 patients $(49.1 \%)$.

3.5. Clinical Outcome. Table 4 shows long term clinical outcome, relapse of disease, and survival.

With a median follow-up of 59 months (range, 6-139 months), five-year overall survival was $74.6 \%$ and pelvic control was $95.8 \%$. Seven patients (4.2\%) developed pelvic recurrence ( 5 isolated and 2 with synchronous metastatic disease) and $22(12.6 \%)$ distant metastases alone. Mandard TRG3-5 (bad responders) is associated with adverse prognosis.

3.6. Predictive Factors of Mandard Response. A logistic regression analysis was used to assess the independent significance of pre- and on-treatment variables as predictive factors of Mandard good responders (TRG1-2). Table 5 shows the 11 variables with predictive value for Mandard response in univariate analysis.

All were related with tumor downstaging and/or with tumor CRT response except the tumor anal distance. The other variables of Tables 1, 2, and 3 have no significant predictive value.

The eleven variables were entered into the Cox-stepwise likelihood ratio. The selection was based upon their statistical significance in univariate analysis and their potential clinical interaction. In the multivariate analysis, after adjustment for the effects of the other variables, tumor with moderated or poor differentiation grade, scarce or moderated necrosis, and $>9.5$ mitosis number for 10 high-powered fields were more likely to present a bad response (Table 6). 
TABLE 4: Clinical long term outcome (follow-up median: 59 months; range 6-139).

\begin{tabular}{lc}
\hline Variable & \\
\hline Overall disease recurrence & $29(17.3 \%)$ \\
$\quad$ Local & $5(3 \%)$ \\
$\quad$ Distant & $22(13.2 \%)$ \\
$\quad$ Local and distant & $2(1.1 \%)$ \\
Five-year overall survival & $74.6 \%(\mathrm{se}=3.8 \%)$ \\
$\begin{array}{l}\text { Five-year overall survival for "good } \\
\text { responders"* } \\
\begin{array}{l}\text { Five-year overall survival for "bad } \\
\text { responders"* }\end{array}\end{array}$ \\
\hline
\end{tabular}

${ }^{*} p<0.001$ (log rank test).

\section{Discussion}

Neoadjuvant CRT followed by curative surgery and adjuvant chemotherapy is used in advanced rectal cancer and the prognosis depends essentially on tumor response. This kind of treatment allows a better pelvic disease control, but better prognosis is achieved only in good responders [2].

The variety of tumor responses has increased the need to find a useful predictive model for the response to CRT in order to identify patients who will really benefit from this multimodal treatment.

In our previous studies Mandard TRG system proved to be fairly accurate, and patients division in good (TRG1-2) and bad responders (TRG3-5) proved effective [10,14]. These results have been confirmed by other groups $[2,12,15,16]$. Based on those results it would be important to analyze potential clinical and pathological factors that influence Mandard response.

The influence of clinical parameters in tumor response has been widely studied. In some studies, tumor size, tumor circumferential extent, poor differentiation, mucinous tumor, distance from anal verge, clinical $\mathrm{T}$ stage, nodal clinical stage, pretreatment carcinoembryonic antigen (CEA) level, and/or interval of time between surgery and radiotherapy completion were associated with CRT tumor response [1726]. Magnetic resonance imaging (MRI) and positron emission tomography-computed tomography (PET-CT) also may be useful to predict the response at early stages [27-33]. However, no clinical parameters with prediction value of CRT response have been consistently identified. The results are often different $[19,23,29,34,35]$. In our series, distance from anal verge is the only clinical parameter in univariate analysis with predictive value for Mandard response: distal tumors ( $\leq 6 \mathrm{~cm}$ from anal verge) show a better response according to Mandard. This result is concordant with that described by Das et al. [18]. The opposite is described by Restivo et al. [36]. The delay of surgery after radiotherapy completion from 8 to 12 weeks seems to increase tumor necrosis grade and pathological complete response (ypCR) rate up to 30 to $40 \%$ [37-40]. We have $18.5 \%$ of ypCR but we cannot study this variable because our patients were operated on average within
8 weeks after radiotherapy conclusion. Pretreatment CEA level is probably the most cited clinical parameter as having tumor response predictive value $[18,20,21,41-44]$. On our study CEA level, other clinical parameters and the biopsy characteristics analyzed did not predict tumor response to nCRT. Biopsy obtained data, namely, differentiation grade, mitotic index, necrosis, inflammatory, and desmoplastic reaction amount, had no utility to recognize or predict tumor behavior. Tumor hypoxia and proliferative cell activity reduce the effectiveness of both radiation therapy and chemotherapy and are a well-known risk factor for tumor radioresistance. So, it was expected that necrosis, grade, and mitotic number in 10 high-power fields could give any indication of CRT tumor response. However in our series it did not occur with a statistically significant correlation. This may be due to several reasons. The amount of tumor in the biopsy is a very small percentage of the total volume of the whole tumor and, as tumors are heterogeneous, biopsy may not be representative of tumor biology. On the other hand, it suggests that other biologic indicators of response must be investigated, because the ones included in this study are not important for this purpose. Finally it must be remembered that tumor is not the unique actor in the process; local and systemic response of the host, immune, and inflammatory factors also need to be considered.

In our data, all predictive factors found are one way or another related with tumor downstaging and/or nCRT tumor response pathological variables found after treatment. This is an expected result. Pathological TNM stage, tumor downstaging, and CMR of surgical specimen are reflected on nCRT tumor response and are considered in many studies prognostic factors of survival [45-49]. In our study the posttreatment differentiation, feasibility, and proliferative activity of tumor cells show greater impact as predictors of Mandard response (more than pathological TNM stage or tumor downstaging). The presence of accentuated necrosis, mitosis number $>9.5$ for 10 high-powered fields, and moderate or poorly differentiated grade in resected specimen had a predictive value for Mandard response of $85 \%$. Those variables had a significant predictive value of Mandard response in multivariate analysis.

Thus, the only process for assessing nCRT response is obtained from posttreatment variables. The absence of reliable clinical predictors of response to CRT emphasizes the need to find other factors able to predict response and thus individualize the treatment approach in LARC. On this series the Mandard grade response proved useful to modify the adjuvant treatment plan in patients who were "bad responders" into a more aggressive treatment.

\section{Conclusions}

Mandard system provides an important tool for survival analysis. None of the clinical or the biopsy characteristics assessed had a predictive value of Mandard response, except the distance from anal verge. Only postoperative pathological parameters related with tumor response to chemoradiotherapy have predictive value for Mandard response. 
TABLE 5: Predictive value of clinical and pathological characteristics to Mandard response (univariate analysis).

\begin{tabular}{|c|c|c|c|c|}
\hline Variables & $n$ & Bad responders \% (Mandard TRG 3-5) & Odds ratio & $p$ \\
\hline \multicolumn{5}{|c|}{ Distance from anal verge } \\
\hline$\leq 6 \mathrm{~cm}$ & 86 & 58.0 & 1.00 & 0.017 \\
\hline$>6 \mathrm{~cm}$ & 81 & 39.5 & $2.11(1.14-3.92)$ & \\
\hline \multicolumn{5}{|c|}{ Mitosis number in resected specimen } \\
\hline$\leq 9.5$ & 73 & 32.9 & 1.00 & $<0.001$ \\
\hline$\geq 9.6$ & 68 & 77.9 & $7.21(3.40-15.32)$ & \\
\hline \multicolumn{5}{|c|}{ Necrosis grade in resected specimen } \\
\hline Scarce & 27 & 77.8 & $10.36(3.76-28.57)$ & $<0.001$ \\
\hline Moderate & 38 & 92.1 & $34.53(9.76-122.41)$ & $<0.001$ \\
\hline Marked & 99 & 25.3 & 1.00 & $<0.001$ \\
\hline \multicolumn{5}{|l|}{ CRM distance } \\
\hline$\geq 2 \mathrm{~mm}$ & 159 & 45.9 & 1.00 & 0.003 \\
\hline$<2$ and $>1 \mathrm{~mm}$ & 8 & 100 & $2.18(1.84-2.58)$ & \\
\hline \multicolumn{5}{|l|}{ ypT stage } \\
\hline $0-1$ & 38 & 7.9 & 1.00 & $<0.001$ \\
\hline $2-4$ & 129 & 60.5 & $17.84(5.21-61.09)$ & \\
\hline \multicolumn{5}{|l|}{ ypN stage } \\
\hline 0 & 110 & 32.7 & 1.00 & \\
\hline $1-2$ & 57 & 78.9 & $7.71(3.64-16.34)$ & $<0.001$ \\
\hline \multicolumn{5}{|l|}{ Pathological stage } \\
\hline $0-\mathrm{I}$ & 58 & 15.5 & 1.00 & $<0.001$ \\
\hline II & 58 & 55.2 & $6.70(2.78-16.14)$ & $<0.001$ \\
\hline III & 51 & 78.4 & $19.80(7.47-52.48)$ & $<0.001$ \\
\hline \multicolumn{5}{|l|}{ T downstaging } \\
\hline Yes & 67 & 23.9 & 1.00 & $<0.001$ \\
\hline No & 100 & 65.0 & 5.92 & \\
\hline \multicolumn{5}{|c|}{ Pathological TNM downstaging } \\
\hline Yes & 96 & 34.4 & 1.00 & $<0.001$ \\
\hline No & 70 & 67.1 & 3.90 & \\
\hline \multicolumn{5}{|c|}{ Reduction of mitosis number } \\
\hline Yes & 83 & 41.0 & 1.00 & $<0.001$ \\
\hline No & 52 & 73.1 & $3.91(1.84-8.30)$ & \\
\hline \multicolumn{5}{|c|}{ Differentiation grade in resected specimen } \\
\hline $0+1$ & 52 & 15.4 & 1.00 & $<0.001$ \\
\hline $2+3$ & 115 & 63.5 & 9.56 & \\
\hline
\end{tabular}

TABle 6: Multivariate stepwise model-dependent variable Mandard response: “0” good response and "1" bad response.

\begin{tabular}{lcc}
\hline Variable & Odds ratio & Confidence interval 95\% \\
\hline Differentiation grade in resected specimen & & \\
$\quad 0+1$ & 1.00 & $3.38-32.30$ \\
$2+3$ & 10.45 & \\
Necrosis grade in resected specimen & & \\
$\quad$ Marked & 1.00 & $3.04-25.54$ \\
$\quad$ Scarce/moderate & 8.82 & \\
Mitosis number in resected specimen & & \\
$\quad \leq 9.5$ & 1.00 & $1.52-10.90$ \\
$\geq 9.6$ & 4.07 & 0.001 \\
\hline
\end{tabular}


Based on these results it is not yet possible to identify the group of patients who truly benefits from neoadjuvant CRT, but we can identify the group of patients (the Mandard bad responders) that will benefit with a more aggressive adjuvant treatment.

\section{Conflict of Interests}

The authors of the paper do not have any financial relation or any financial interest with the subject matter or materials discussed in this paper.

\section{References}

[1] R. Sauer, T. Liersch, S. Merkel et al., "Preoperativeversus postoperative chemoradiotherapy for locally advanced rectal cancer: results of the German CAO/ARO/AIO -94 randomized phase III trial afteramedian follow-up of 11 years," Journal of Clinical Oncology, vol. 30, no. 16, pp. 1926-1933, 2012.

[2] Y.-C. Lee, C.-C. Hsieh, and J.-P. Chuang, "Prognostic significance of partial tumor regression after preoperative chemoradiotherapy for rectal cancer: a meta-analysis," Diseases of the Colon \& Rectum, vol. 56, no. 9, pp. 1093-1101, 2013.

[3] S. T. Martin, H. M. Heneghan, and D. C. Winter, "Systematic review and meta-analysis of outcomes following pathological complete response to neoadjuvant chemoradiotherapy for rectal cancer," British Journal of Surgery, vol. 99, no. 7, pp. 918-928, 2012.

[4] C. Rodel, P. Martus, and T. Papadoupolos, "Prognostic significance of tumor regression after preoperative chemoradiotherapy for rectal cancer," Journal of Clinical Oncology, vol. 23, no. 34, pp. 8688-8696, 2005.

[5] P. Roy, S. Serra, E. Kennedy, and R. Chetty, “The prognostic value of grade of regression and oncocytic change in rectal adenocarcinoma treated with neo-adjuvant chemoradiotherapy," Journal of Surgical Oncology, vol. 105, no. 2, pp. 130-134, 2012.

[6] L. Topova, G. Hellmich, E. Puffer et al., "Prognostic value of tumor response to neoadjuvant therapy in rectal carcinoma," Diseases of the Colon and Rectum, vol. 54, no. 4, pp. 401-411, 2011.

[7] F. M. Vecchio, V. Valentini, B. D. Minsky et al., "The relationship of pathologic tumor regression grade (TRG) and outcomes after preoperative therapy in rectal cancer," International Journal of Radiation Oncology, Biology, Physics, vol. 62, pp. 752-760, 2005.

[8] J. M. D. Wheeler, E. Dodds, B. F. Warren et al., "Preoperative chemoradiotherapy and total mesorectal excision surgery for locally advanced rectal cancer: correlation with rectal cancer regression grade," Diseases of the Colon \& Rectum, vol. 47, no. 12, pp. 2025-2031, 2004.

[9] A.-M. Mandard, F. Dalibard, J.-C. Mandard et al., "Pathologic assessment of tumor regression after preoperative chemoradiotherapy of esophageal carcinoma: clinicopathologic correlations," Cancer, vol. 73, no. 11, pp. 2680-2686, 1994.

[10] M. D. Santos, C. Silva, A. Rocha, E. Matos, C. Nogueira, and C. Lopes, "Prognostic value of Mandard and Dworak tumor regression grading in rectal cancer: study of a single tertiary center," ISRN Surgery, vol. 2014, Article ID 310542, 8 pages, 2014.

[11] A. C. Bateman, E. Jaynes, and A. R. Bateman, "Rectal cancer staging post neoadjuvant therapy-how should the changes be assessed?" Histopathology, vol. 54, no. 6, pp. 713-721, 2009.
[12] D. Beddy, J. M. P. Hyland, D. C. Winter et al., "A simplified tumor regression grade correlates with survival in locally advanced rectal carcinoma treated with neoadjuvant chemoradiotherapy," Annals of Surgical Oncology, vol. 15, no. 12, pp. 3471-3477, 2008.

[13] T. P. MacGregor, T. S. Maughan, and R. A. Sharma, "Pathological grading of regression following neoadjuvant chemoradiation therapy: the clinical need is now," Journal of Clinical Pathology, vol. 65, no. 10, pp. 867-871, 2012.

[14] M. D. Santos, C. Silva, A. Rocha, E. Matos, C. Nogueira, and C. Lopes, "Tumor regression grades: can they influence rectal cancer therapy decision tree?” International Journal of Surgical Oncology, vol. 2013, Article ID 572149, 8 pages, 2013.

[15] J. Suárez, R. Vera, E. Balén et al., "Pathologic response assessed by Mandard grade is a better prognostic factor than down staging for disease-free survival after preoperative radiochemotherapy for advanced rectal cancer," Colorectal Disease, vol. 10, no. 6, pp. 563-568, 2008.

[16] M. Huebner, B. G. Wolff, T. C. Smyrk, J. Aakre, and D. W. Larson, "Partial pathologic response and nodal status as most significant prognostic factors for advanced rectal cancer treated with preoperative chemoradiotherapy," World Journal of Surgery, vol. 36, no. 3, pp. 675-683, 2012.

[17] B. Farnault, L. Moureau-Zabotto, C. de Chaisemartin et al., "Predictive factors of tumour response after neoadjuvant chemoradiation for locally advanced rectal cancer and correlation of these factors with survival," Cancer/Radiothérapie, vol. 15, no. 4, pp. 279-286, 2011.

[18] P. Das, J. M. Skibber, M. A. Rodrigues-Bigas et al., "Predictors of tumor response and downstaging in patients who receive preoperative chemoradiation for rectal cancer," Cancer, vol. 109, no. 9, pp. 1750-1755, 2007.

[19] K.-L. Yang, S.-H. Yang, W.-Y. Liang et al., "Carcinoembryonic antigen (CEA) level, CEA ratio, and treatment outcome of rectal cancer patients receiving pre-operative chemoradiation and surgery," Radiation Oncology, vol. 8, article 43, 2013.

[20] H. Yan, R. Wang, K. Zhu et al., "Predictors of sensitivity to preoperative chemoradiotherapy of rectal adenocarcinoma," Tumori, vol. 97, no. 6, pp. 717-723, 2011.

[21] S.-G. Yeo, D. Y. Kim, H. J. Chang et al., "Reappraisal of pretreatment carcinoembryonic antigen in patients with rectal cancer receiving preoperative chemoradiotherapy," Tumori, vol. 99, no. 1, pp. 93-99, 2013.

[22] D. Tural, F. Selcukbiricik, M. A. Özturk et al., "The relation between pathological complete response and clinical outcome in patients with rectal cancer," Hepatogastroenterology, vol. 60, no. 126, pp. 1365-1370, 2013.

[23] H.-Z. Qiu, B. Wu, Y. Xiao, and G.-L. Lin, "Combination of differentiation and $\mathrm{T}$ stage can predict unresponsiveness to neoadjuvant therapy for rectal cancer," Colorectal Disease, vol. 13, no. 12, pp. 1353-1360, 2011.

[24] K. Oberholzer, M. Menig, A. Kreft et al., "Rectal cancer: mucinous carcinoma on magnetic resonance imaging indicates poor response to neoadjuvant chemoradiation," International Journal of Radiation Oncology, Biology, Physics, vol. 82, no. 2, pp. 842-848, 2012.

[25] H. Tulchinsky, E. Shmueli, A. Figer, J. M. Klausner, and M. Rabau, "An interval $>7$ weeks between neoadjuvant therapy and surgery improves pathologic complete response and diseasefree survival in patients with locally advanced rectal cancer," Annals of Surgical Oncology, vol. 15, no. 10, pp. 2661-2667, 2008. 
[26] J. W. Huh, H. R. Kim, and Y. J. Kim, "Clinical prediction of pathological complete response after preoperative chemoradiotherapy for rectal cancer," Diseases of the Colon and Rectum, vol. 56, no. 6, pp. 698-703, 2013.

[27] D. W. Dietz, F. Dehdashti, P. W. Grigsby et al., "Tumor hypoxia detected by positron emission tomography with 60Cu-ATSM as a predictor of response and survival in patients undergoing neoadjuvant chemoradiotherapy for rectal carcinoma: a pilot study," Diseases of the Colon \& Rectum, vol. 51, no. 11, pp. 1641$1648,2008$.

[28] X. Meng, Z. Huang, R. Wang, and J. Yu, "Prediction of response to preoperative chemoradiotherapy in patients with locally advanced rectal cancer," BioScience Trends, vol. 8, no. 1, pp. 1123, 2014.

[29] J. W. Kim, H. C. Kim, J. W. Park et al., "Predictive value of ${ }^{18}$ FDG PET-CT for tumour response in patients with locally advanced rectal cancer treated by preoperative chemoradiotherapy," International Journal of Colorectal Disease, vol. 28, no. 9, pp. 12171224, 2013.

[30] H. Everaert, A. Hoorens, C. Vanhove et al., "Prediction of response to neoadjuvant radiotherapy in patients with locally advanced rectal cancer by means of sequential 18FDG-PET," International Journal of Radiation Oncology Biology Physics, vol. 80, no. 1, pp. 91-96, 2011.

[31] D. Ippolito, L. Monguzzi, L. Guerra et al., "Response to neoadjuvant therapy in locally advanced rectal cancer: assessment with diffusion-weighted MR imaging and 18FDG PET/CT," Abdominal Imaging, vol. 37, no. 6, pp. 1032-1040, 2012.

[32] S. H. Jung, S. H. Heo, J. W. Kim et al., "Predicting response to neoadjuvant chemoradiation therapy in locally advanced rectal cancer: diffusion-weighted 3 tesla MR imaging," Journal of Magnetic Resonance Imaging, vol. 35, no. 1, pp. 110-116, 2012.

[33] S. Shanmugan, R. Arrangoiz, J. R. Nitzkorski et al., "Predicting pathological response to neoadjuvant chemoradiotherapy in locally advanced rectal cancer using ${ }^{18}$ FDG-PET/CT," Annals of Surgical Oncology, vol. 19, no. 7, pp. 2178-2185, 2012.

[34] D. J. Kim, J. H. Kim, J. S. Lim et al., "Restaging of rectal cancer with MR imaging after concurrent chemotherapy and radiation therapy," Radiographics, vol. 30, no. 2, pp. 503-516, 2010.

[35] C. H. Choi, W. D. Kim, S. J. Lee, and W. Y. Park, "Clinical predictive factors of pathologic tumor response after preoperative chemoradiotherapy in rectal cancer," Radiation Oncology Journal, vol. 30, no. 3, pp. 99-107, 2012.

[36] A. Restivo, L. Zorcolo, I. M. F. Cocco et al., "Elevated CEA levels and low distance of the tumor from the anal verge are predictors of incomplete response to chemoradiation in patients with rectal cancer," Annals of Surgical Oncology, vol. 20, no. 3, pp. 864-871, 2013.

[37] L. F. de Campos-Lobato, D. P. Geisler, A. da Luz Moreira, L. Stocchi, D. Dietz, and M. F. Kalady, "Neoadjuvant therapy for rectal cancer: the impact of longer interval between chemoradiation and surgery," Journal of Gastrointestinal Surgery, vol. 15, no. 3, pp. 444-450, 2011.

[38] A. Habr-Gama, R. O. Perez, I. Proscurshim et al., "Interval between surgery and neoadjuvant chemoradiation therapy for distal rectal cancer: does delayed surgery have an impact on outcome?" International Journal of Radiation Oncology, Biology, Physics, vol. 71, no. 4, pp. 1181-1188, 2008.

[39] J. Garcia-Aguilar, D. D. Smith, K. Avila, E. K. Bergsland, P. Chu, and R. M. Krieg, "Optimal timing of surgery after chemoradiation for advanced rectal cancer: preliminary results of a multicenter, nonrandomized phase II prospective trial," Annals of Surgery, vol. 254, no. 1, pp. 97-102, 2011.

[40] C. G. Willett and B. G. Czito, "Impact of time duration after neoadjuvant therapy to surgery on response and outcome in rectal cancer patients," Annals of Surgical Oncology, vol. 15, no. 10, pp. 2636-2638, 2008.

[41] Y.-J. Kim, S. C. Park, D. Y. Kim et al., "No correlation between pretreatment serum CEA levels and tumor volume in locally advanced rectal cancer patients," Clinica Chimica Acta, vol. 413, no. 3-4, pp. 511-515, 2012.

[42] L. Moureau-Zabotto, B. Farnault, C. de Chaisemartin et al., "Predictive factors of tumor response after neoadjuvant chemoradiation for locally advanced rectal cancer," International Journal of Radiation Oncology Biology Physics, vol. 80, no. 2, pp. 483-491, 2011.

[43] D. Tural, F. Selcukbiricik, M. A. Özturk et al., "The relation between pathological complete response and clinical outcome in patients with rectal cancer," Hepato-Gastroenterology, vol. 60, no. 126, pp. 1365-1370, 2013.

[44] U. Wallin, D. Rothenberger, A. Lowry, R. Luepker, and A. Mellgren, "CEA - a predictor for pathologic complete response after neoadjuvant therapy for rectal cancer," Diseases of the Colon \& Rectum, vol. 56, no. 7, pp. 859-868, 2013.

[45] S.-G. Yeo, D. Y. Kim, T. H. Kim et al., "Pathologic complete response of primary tumor following preoperative chemoradiotherapy for locally advanced rectal cancer: long-term outcomes and prognostic significance of pathologic nodal status (KROG 09-01)," Annals of Surgery, vol. 252, no. 6, pp. 998-1004, 2010.

[46] S.-G. Yeo, D. Y. Kim, J. W. Park et al., "Stage-to-stage comparison of preoperative and postoperative chemoradiotherapy for T3 mid or distal rectal cancer," International Journal of Radiation Oncology Biology Physics, vol. 82, no. 2, pp. 856-862, 2012.

[47] A. K. P. Chan, A. Wong, D. Jenken, J. Heine, D. Buie, and D. Johnson, "Posttreatment TNM staging is a prognostic indicator of survival and recurrence in tethered or fixed rectal carcinoma after preoperative chemotherapy and radiotherapy," International Journal of Radiation Oncology, Biology, Physics, vol. 61, no. 3, pp. 665-677, 2005.

[48] H. M. Quah, J. F. Chou, M. Gonen et al., "Pathologic stage is most prognostic of disease-free survival in locally advanced rectal cancer patients after preoperative chemoradiation," Cancer, vol. 113, pp. 57-64, 2008.

[49] P. Luna-Pérez, E. Bustos-Cholico, I. Alvarado et al., "Prognostic significance of circumferential margin involvement in rectal adenocarcinoma treated with preoperative chemoradiotherapy and low anterior resection," Journal of Surgical Oncology, vol. 90, no. 1, pp. 20-25, 2005. 


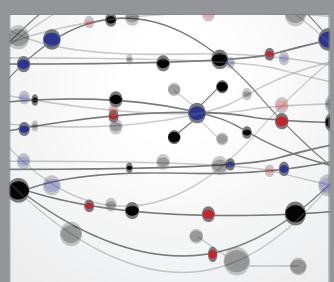

The Scientific World Journal
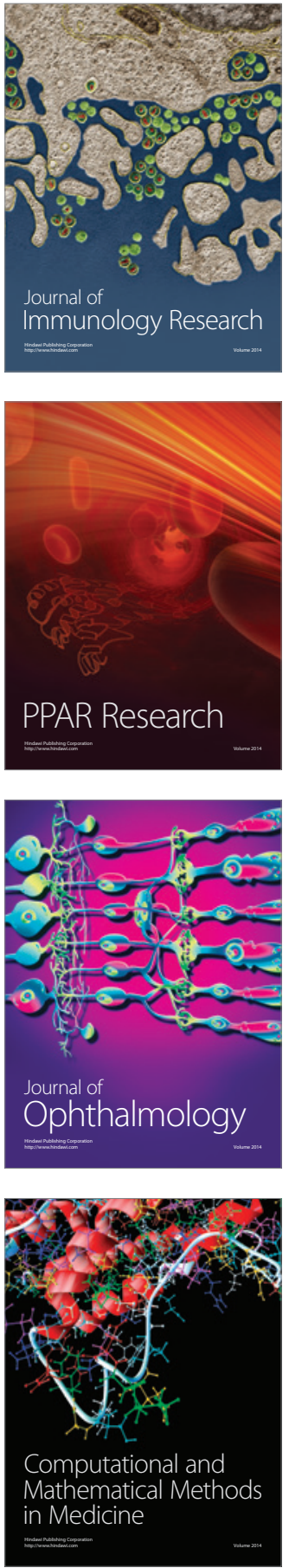

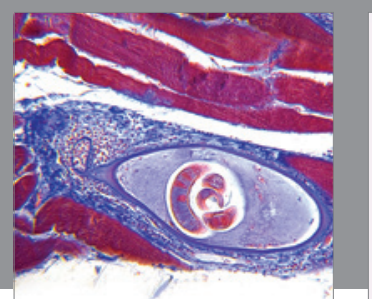

Gastroenterology Research and Practice

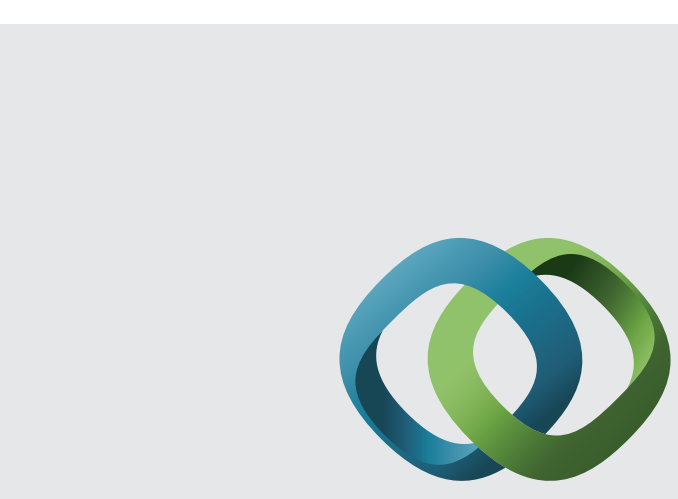

\section{Hindawi}

Submit your manuscripts at

http://www.hindawi.com
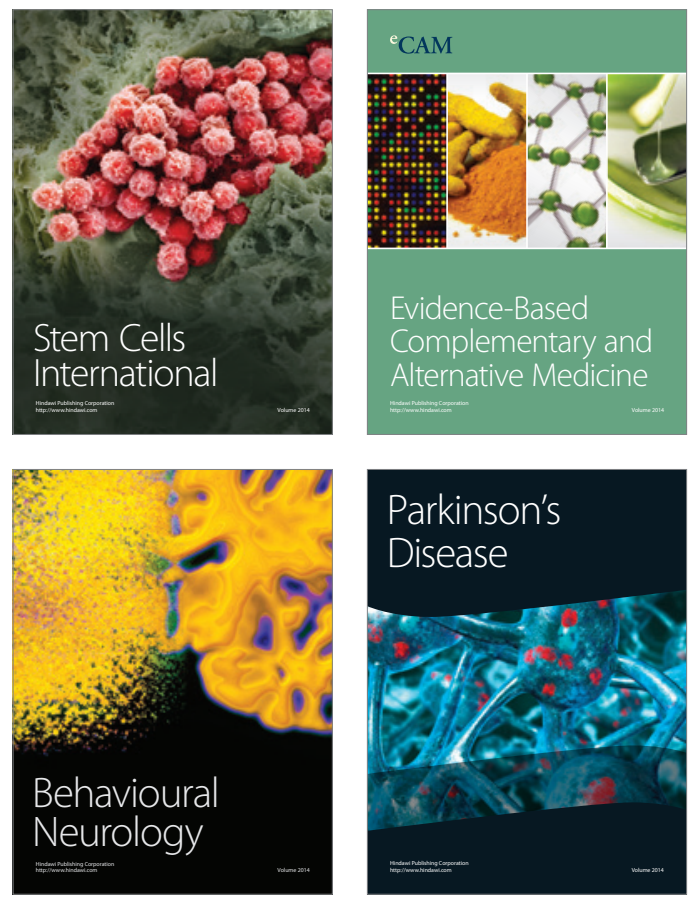
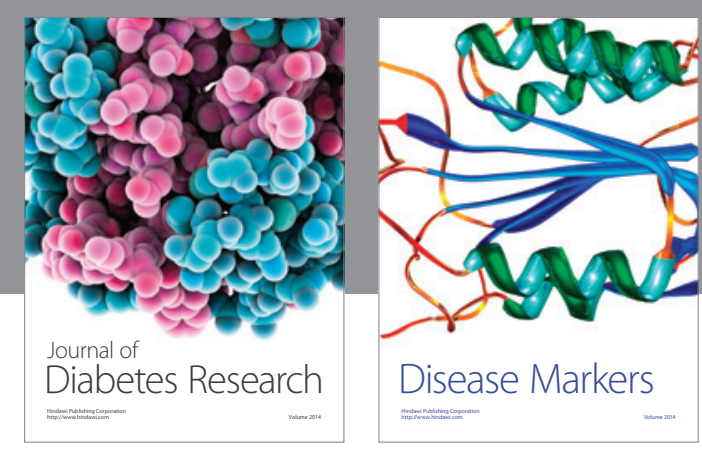

Disease Markers
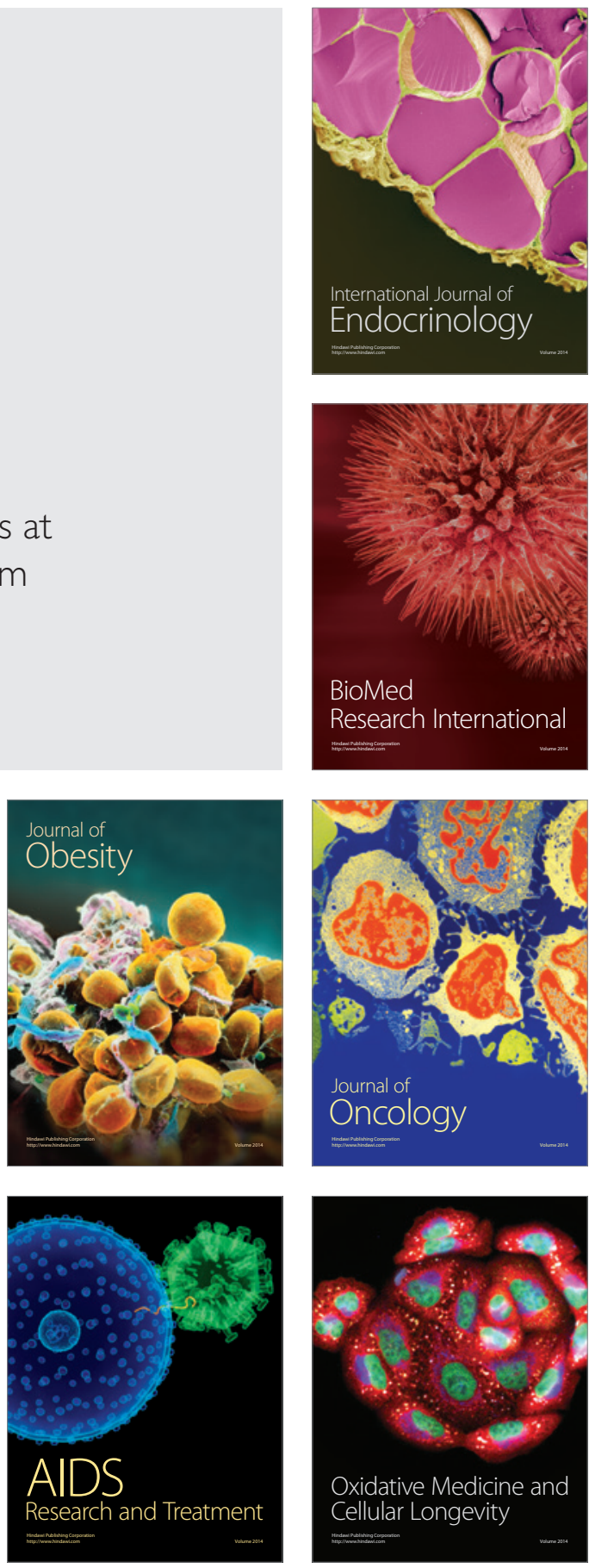